\title{
Isolated nocturnal and isolated daytime hypertension associate with altered cardiovascular morphology and function in children with chronic kidney disease: findings from the Cardiovascular Comorbidity in Children with Chronic Kidney Disease study
}

\author{
Düzova, Ali ; Karabay Bayazit, Aysun ; Canpolat, Nur ; et al ; Laube, Guido
}

\begin{abstract}
INTRODUCTION Prevalence of isolated nocturnal hypertension (INH) and isolated daytime hypertension (IDH) is around $10 \%$ in adults. Data in children, especially in chronic kidney disease (CKD), are lacking. The aim of this cross-sectional multicenter cohort study was to define the prevalence of INH and IDH and its association with cardiovascular morphology and function, that is, pulse wave velocity (PWV), carotid intima-media thickness (cIMT), or left ventricular mass index (LVMI) in children with CKD. METHODS Ambulatory blood pressure (BP) monitoring profiles were analyzed in 456 children with CKD stages III-V participating in the Cardiovascular Comorbidity in Children with Chronic Kidney Disease Study (64.3\% males, $71.3 \%$ congenital anomaly of the kidney and urinary tract, age $12.5 \pm$ 3.2 years, estimated glomerular filtration rate $29 \pm 12 \mathrm{ml} / \mathrm{min}$ per $1.73 \mathrm{~m}$ ). Baseline PWV, cIMT, and LVMI were compared in normotension, INH, IDH, or sustained 24-h hypertension. RESULTS Prevalence of sustained hypertension was $18.4 \%$, of INH $13.4 \%$, and of IDH 3.7\%. PWV SDS (SD score) and cIMT SDS were significantly higher in sustained hypertension and INH, and PWV SDS was significantly higher in IDH, compared with normotension. LVMI was significantly increased in sustained hypertension, but not in INH or IDH. Determinants of INH were smallness for gestational age, older age, higher height SDS and parathyroid hormone, and shorter duration of CKD. In logistic regression analysis, day/night-time hypertension or ambulatory BP monitoring pattern (normal, INH, IDH, sustained hypertension) were independently associated with cardiovascular outcome measures: elevated night-time BP was associated with increased cIMT, PWV, and left ventricular hypertrophy; INH was associated with cIMT. CONCLUSION INH is present in almost one out of seven children with predialysis CKD; INH and nocturnal hypertension in general are associated with alterations of arterial morphology and function.
\end{abstract}

DOI: https://doi.org/10.1097/HJH.0000000000002160

Posted at the Zurich Open Repository and Archive, University of Zurich

ZORA URL: https://doi.org/10.5167/uzh-182481

Journal Article

Published Version

Originally published at:

Düzova, Ali; Karabay Bayazit, Aysun; Canpolat, Nur; et al; Laube, Guido (2019). Isolated nocturnal and isolated daytime hypertension associate with altered cardiovascular morphology and function in children with chronic kidney disease: findings from the Cardiovascular Comorbidity in Children with Chronic Kidney Disease study. Journal of Hypertension, 37(11):2247-2255.

DOI: https://doi.org/10.1097/HJH.0000000000002160 
Isolated nocturnal and isolated daytime hypertension associate with altered cardiovascular morphology and function in children with chronic kidney disease: findings from the Cardiovascular Comorbidity in Children with Chronic Kidney Disease study

\author{
Ali Düzova ${ }^{a}$, Aysun Karabay Bayazit ${ }^{b}$, Nur Canpolat ${ }^{c}$, Anna Niemirska $^{d}$, Ipek Kaplan Bulut ${ }^{\mathrm{e}}$, \\ Karolis Azukaitis ${ }^{f}$, Tevfik Karagoz ${ }^{g}$, Berna Oguz ${ }^{\text {, }}$, Sevcan Erdemi, Ali Anarat ${ }^{b}$, Bruno Ranchin ${ }^{j}$,

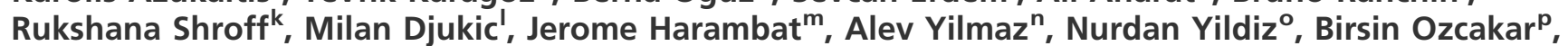 \\ Anja Büscher ${ }^{q}$, Francesca Lugani ${ }^{r}$, Simone Wygodas $^{\mathrm{s}}$, Sibylle Tschumi ${ }^{\mathrm{t}}$, Ariane Zaloszyc ${ }^{\mathrm{u}}$, \\ Augustina Jankauskienev ${ }^{v}$, Guido Laubew ${ }^{w}$ Matthias Galiano ${ }^{x}$, Marietta Kirchner ${ }^{y}$, Uwe Querfeld ${ }^{z}$, \\ Anette Melk $^{\text {aa }}$, Franz Schaefer ${ }^{\text {bb }}$, and Elke Wühl ${ }^{\text {bb }}$, for the 4C Study Consortium
}

\begin{abstract}
Introduction: Prevalence of isolated nocturnal hypertension (INH) and isolated daytime hypertension (IDH) is around $10 \%$ in adults. Data in children, especially in chronic kidney disease (CKD), are lacking. The aim of this cross-sectional multicenter cohort study was to define the prevalence of INH and IDH and its association with cardiovascular morphology and function, that is, pulse wave velocity (PWV), carotid intima-media thickness (cIMT), or left ventricular mass index (LVMI) in children with CKD.
\end{abstract}

Methods: Ambulatory blood pressure (BP) monitoring profiles were analyzed in 456 children with CKD stages III-V participating in the Cardiovascular Comorbidity in Children with Chronic Kidney Disease Study (64.3\% males, $71.3 \%$ congenital anomaly of the kidney and urinary tract, age $12.5 \pm 3.2$ years, estimated glomerular filtration rate $29 \pm 12 \mathrm{ml} / \mathrm{min}$ per $1.73 \mathrm{~m}^{2}$ ). Baseline PWV, cIMT, and LVMI were compared in normotension, INH, IDH, or sustained 24-h hypertension.

Results: Prevalence of sustained hypertension was 18.4\%, of INH 13.4\%, and of IDH 3.7\%. PWV SDS (SD score) and CIMT SDS were significantly higher in sustained hypertension and INH, and PWV SDS was significantly higher in IDH, compared with normotension. LVMI was significantly increased in sustained hypertension, but not in INH or IDH. Determinants of INH were smallness for gestational age, older age, higher height SDS and parathyroid hormone, and shorter duration of CKD. In logistic regression analysis, day/night-time hypertension or ambulatory BP monitoring pattern (normal, INH, IDH, sustained hypertension) were independently associated with cardiovascular outcome measures: elevated night-time BP was associated with increased CIMT, PWV, and left ventricular hypertrophy; INH was associated with cIMT.
Conclusion: INH is present in almost one out of seven children with predialysis CKD; INH and nocturnal hypertension in general are associated with alterations of arterial morphology and function.

Keywords: ambulatory blood pressure monitoring, carotid intima-media thickness, chronic kidney disease, isolated daytime hypertension, isolated nocturnal hypertension, left ventricular hypertrophy, left ventricular mass index, pulse wave velocity

Journal of Hypertension 2019, 37:2247-2255

aDivision of Pediatric Nephrology, Hacettepe University Faculty of Medicine, Ankara, ${ }^{b}$ Cukurova University Faculty of Medicine, Adana, 'Istanbul University Cerrahpasa Medical Faculty, Istanbul, Turkey, 'The Children's Memorial Health Institute, Warsaw, Poland, 'Ege University Faculty of Medicine, Izmir, Turkey, ${ }^{f} C$ linic of Pediatrics, Faculty of Medicine, Vilnius, Lithuania, ${ }^{9}$ Division of Pediatric Cardiology, hDepartment of Radiology, Hacettepe University Faculty of Medicine, Ankara, 'Division of Pediatric Cardiology, Cukurova University Faculty of Medicine, Adana, Turkey, ${ }^{\mathrm{H} H o s p i c e s}$ Civils de Lyon, Bron, France, ${ }^{k}$ Great Ormond Street Hospital for Children, London, UK, 'School of Medicine, University of Belgrade, Belgrade, Serbia, mPediatric Nephrology Unit, Bordeaux University Hospital, Bordeaux, France, "Istanbul University Istanbul Medical Faculty, ${ }^{\circ}$ Marmara University Faculty of Medicine, Istanbul, PDivision of Pediatric Nephrology and Rheumatology, Department of Pediatrics, Ankara University Medical School, Ankara, Turkey, 9University Children's Hospital, Pediatrics 2, Pediatric Nephrology, University of Duisburg-Essen, Essen, Germany, 'Division of Nephrology

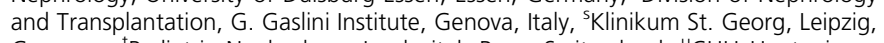
Germany, 'Pediatric Nephrology, Inselspital, Bern, Switzerland, "CHU Hautepierre, Strasbourg, France, "Institute of Clinical Medicine, Vilnius University, Vilnius, Lithuania, "wephrology Unit, University Children's Hospital, Zürich, Switzerland, ${ }^{x}$ Department of Pediatrics and Adolescent Medicine, University of Erlangen-Nuremberg, Erlangen, ${ }^{\mathrm{y}}$ Institute of Medical Biometry and Informatics, Heidelberg, ${ }^{\mathrm{z}}$ Charité

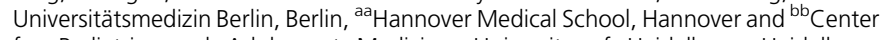
for Pediatrics and Adolescent Medicine, University of Heidelberg, Heidelberg, Germany

Correspondence to Ali Düzova, Division of Pediatric Nephrology, Department of Pediatrics, Hacettepe University Faculty of Medicine, Sihhiye, 06100 Ankara, Turkey. E-mail: aduzova@hacettepe.edu.tr

Received 7 July 2018 Revised 11 April 2019 Accepted 26 April 2019

J Hypertens 37:2247-2255 Copyright (C) 2019 Wolters Kluwer Health, Inc. All rights reserved.

DOI:10.1097/HJH.0000000000002160 


\begin{abstract}
Abbreviations: $A B P M$, ambulatory blood pressure monitoring; BP, blood pressure; CAKUT, congenital anomaly of the kidney and urinary tract; CIMT, carotid intima-media thickness; CKD, chronic kidney disease; eGFR, estimated glomerular filtration rate; ESRD, end-stage renal disease; HTN, hypertension; IDH, isolated daytime hypertension; INH, isolated nocturnal hypertension; LVH, left ventricular hypertrophy; LVM, left ventricular mass; LVMI, left ventricular mass index; PTH, parathyroid hormone; PWV, pulse wave velocity; SDS, standard deviation score; SGA, smallness for gestational age
\end{abstract}

\section{INTRODUCTION}

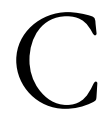
hildren with chronic kidney disease (CKD) are at high risk for cardiovascular morbidity and mortality. Cardiovascular disease accounts for the majority of deaths in adults and approximately one quarter of deaths in children with end-stage renal disease (ESRD) [1]. In young adults with ESRD, cardiovascular mortality is increased up to 1000-fold compared with the general population [2]. Uncontrolled hypertension (HTN) is a risk factor for cardiovascular morbidity and for progression of CKD in adults $[3,4]$.

High blood pressure (BP) is also a predictor of renal disease progression in children with CKD [5]. The prevalence of HTN in children with CKD Stages II-IV is 25-50\% in reports from the North American Pediatric Renal Trials and Collaborative Studies, the CKD in Children (CKiD) Study, and the Effect of Strict Blood Pressure Control and ACE Inhibition on Chronic Renal Failure Progression in Pediatric Patients (ESCAPE) trial group [6-8]. Ambulatory BP monitoring (ABPM) is superior to conventional office or home BP monitoring in assessing BP load and stratifying cardiovascular risk, particularly in CKD patients [9].

Li et al. [10] identified a group of individuals who had abnormally elevated night-time BP but normal daytime BP in ABPM; they defined this BP pattern as isolated nocturnal hypertension (INH). INH was associated with clustering of cardiovascular risk factors, thickening of carotid intimamedia, left ventricular (LV) remodeling and increased arterial stiffness in adult patients with essential HTN or type 2 diabetes mellitus who had well controlled self-measured home daytime BP [10-12]. In a population-based cohort study in African-American adults, INH was associated with increased LV mass [13].

In a meta-analysis of 8711 adults from 10 populations, INH predicted cardiovascular outcome in patients who were normotensive based on office or ambulatory daytime BP measurements [14].

In adolescents and young adults with diabetes mellitus type 1, an increase in SBP during sleep preceded the development of microalbuminuria [15]. In a pediatric outpatient clinic, patients (6-18 years old) with persistent masked HTN, defined as an elevated daytime ambulatory $\mathrm{BP}$ in the presence of a normal office BP, had higher LV mass index than normotensive controls [16].

So far, no data have been published on the prevalence of isolated daytime hypertension (IDH) and its impact on cardiovascular health in children.
Here, we aimed to define the prevalence and determinants of INH and IDH, and to evaluate potential associations of INH and IDH with cardiac and vascular morphology and function in children with CKD stages III-V. For this purpose, we analyzed data from the Cardiovascular Comorbidity in Children with Chronic Kidney Disease (4C) Study, a prospective cohort study in nearly 700 European children designed to evaluate the causes and consequences of cardiovascular comorbidity of progressive CKD in childhood and adolescence.

\section{METHODS}

\section{Study population}

The 4C Study enrolled 688 patients, aged 6-17 years, with predialysis CKD stages III-V at 55 pediatric nephrology centers in 12 European countries between October of 2009 and August of 2011 (17). Patients with active systemic vasculitis, diabetes mellitus, renal vascular anomalies, anomalies of the limbs preventing standardized diagnostic procedures, and relevant CKD-unrelated cardiovascular anomalies were excluded. The study was approved by all local ethical committees and conducted according to the Declaration of Helsinki and Good Clinical Practice guidelines. Written informed consent was given by the parents and adolescents, and oral assent by younger children [17].

All children are followed prospectively with 6-monthly clinical assessments, blood and urine collection. ABPM, pulse wave velocity (PWV), carotid intima-media thickness (cIMT), and echocardiographic measurements are performed annually. For the present cross-sectional study, the clinical information and laboratory data at the time of first valid ABPM in predialysis CKD patients were analyzed.

\section{Definitions}

Renal diagnoses were categorized as CAKUT (congenital anomaly of the kidney and urinary tract), glomerulopathies, CKD following acute kidney injury, tubulointerstitial diseases, and other or unknown. Physical activity score was estimated by hours of physical activity $(0,1-2,3-4,>4 \mathrm{~h}$ / week) using a standardized questionnaire $[17,18]$.

Standardized height (height SDS, SD score) and BMI (SDS) were calculated from the WHO Child Growth Standards [19]. SD score (SDS or $z$-score) indicates how many SDs an observation is above or below the mean/median. Office $\mathrm{BP}$ values were standardized according to the algorithm published in the Fourth Report [20].

\section{Laboratory measurements}

A centralized routine laboratory analysis was performed including creatinine, cystatin $\mathrm{C}$, urea, high sensitivity C-reactive protein (hsCRP), albumin, lipid levels, calcium, phosphate, and parathyroid hormone (PTH) as described elsewhere [21]. Hemoglobin ( $\mathrm{Hb}$ ) levels were measured locally. Estimated glomerular filtration rate (eGFR, $\mathrm{ml} / \mathrm{min} /$ $1.73 \mathrm{~m}^{2}$ ) was calculated from creatinine, cystatin C, urea, and height according to a published equation [22]. CKD stage was defined by eGFR according to Kidney Disease Outcomes Quality Initiative guidelines [23]. 


\section{Ambulatory blood pressure monitoring measurements}

ABPM measurements, performed on regular school days, were taken using the Spacelabs Monitor 90207 or 90217 device (Spacelabs Healthcare, Snoqualmie, Washington, USA) with the most appropriate sized cuff applied on the nondominant arm. Readings were taken every 15-20 min during the day and every 30-45 min at night [24]. A valid ABPM profile was defined by at least $18 \mathrm{~h}$ of continuous recording and at least 20 valid daytime (awake) measurements and at least seven at night (asleep) (based on the requirement to have at least $70 \%$ of measurements being obtained every $30 \mathrm{~min}$, or more frequently, throughout the entire 24-h period) and at least one valid reading per hour, including night-time, as a primary criterion for an interpretable study $[25,26]$. A total of 456 profiles of a total of 545 ABPM profiles available (83.7\%) met these quality criteria and were included in the analysis.

The age and sex-specific SDS was calculated for each patient's mean BP according to Wühl et al. [24]. For the purpose of this study ABPM profiles were classified as follows:

(1) Normal ABPM profile (normal-BP) was defined as daytime (0800 to $2000 \mathrm{~h}$ ) and night-time (midnight to $0600 \mathrm{~h}$ ) SBP and DBP less than 95th percentile.

(2) Daytime HTN was defined as daytime SBP and/or DBP at least 95th percentile.

(3) Night-time HTN was defined as night-time SBP and/ or DBP at least 95th percentile.

(4) Sustained hypertension was defined as daytime and night-time SBP and/or DBP at least 95th percentile.

(5) INH was defined as daytime SBP and DBP less than 95th percentile and night-time SBP and/or DBP at least 95 th percentile.

(6) IDH was defined as daytime SBP and/or DBP at least 95th percentile and night-time SBP and DBP less than 95th percentile.

Definitions for white-coat HTN, masked HTN, BP load and dipping are given in Supplementary material [27] (additional definitions for ABPM, http://links.lww.com/ $\mathrm{HJH} / \mathrm{B} 112)$.

\section{Left ventricular mass index}

Standard echocardiographic measurements of the left ventricle were performed. All echocardiographs are evaluated and analyzed by a core lab $[17,18]$. The LV mass (LVM) was determined following the modified Devereux formula [28]. LVM index (LVMI) was calculated according to Chinali et al. [29] $\left[\mathrm{LVMI}=\mathrm{LVM} /\left(\right.\right.$ height $\left.\left.^{2.16}+0.09\right)\right]$. A partition value of $45 \mathrm{~g} / \mathrm{m}^{2.16}$ was defined as the upper normal limit for LVMI.

\section{Carotid intima-media thickness}

Ultrasound examination was performed according to the Mannheim carotid intima-media thickness consensus [30] using an 8-MHz annular array ultrasound imaging system (Siemens Acuson P50 Ultrasound system, Software version 2.1; Siemens Medical Solution USA, Inc. Mountain View, California, USA) with integrated digital image evaluation software (Syngo US Workplace, Siemens Medical Solutions,
USA Inc., Ann Arbor, Michigan, USA). cIMT was obtained either by five averaged measurements on each side or semiautomatically using a digital image evaluation software. All values were transformed to SDS adjusted for height and sex using reference values established by our group [31].

\section{Aortic pulse wave velocity}

Aortic PWV was assessed using the oscillometric Vicorder device (SMT medical, Würzburg, Germany) using the distance from the suprasternal notch to the femoral recording point via the umbilicus as path length. Settings and measurement conditions were as previously reported [32,33]. Description of the measurements are summarized in Supplementary material (Description of aortic PWV measurements, http://links.lww.com/HJH/B112). The measured values were transformed to SDS adjusted for height and sex using reference values established by our group [33].

\section{Statistics}

Normally distributed continuous data were summarized by the mean $\pm S D$, and nonnormally distributed variables were summarized by the median and interquartile range (IQR). Categorical variables were presented as number and percentages and compared by using Chi-square or Fisher's exact test. One-way analysis of variance test or Kruskal-Wallis test was used to compare continuous data between the four ABPM groups, as appropriate; pairwise comparisons ( $t$ test or Mann-Whitney $U$ test) were performed when there was a statistically significant difference between the groups (main effect). Logistic regression analyses were performed to define independent risk factors for INH (reference: normotension); variables associated by univariate analysis at $P$ of 0.20 or less were further entered into the logistic regression analysis. Logistic regression analyses were performed to evaluate whether daytime hypertension, nighttime hypertension, and ABPM patterns (i.e. normal ABPM, INH, IDH, and sustained hypertension) were independently associated with LV hypertrophy, elevated cIMT ( $\geq 95$ th percentile for sex and height) and PWV ( $\geq 95$ th percentile for sex and height). Variables associated by univariate analysis at $P$ of 0.20 or less were further entered into the logistic regression analysis. Two models which included daytime / night-time HTN (Model A) or type of ABPM profiles (Model B) were evaluated. In model A, the presence of daytime (including IDH and sustained hypertension) and night-time HTN (including INH and sustained hypertension) was studied, the reference was normotension during daytime or nighttime, respectively. In model B, the presence of INH, IDH or sustained hypertension was studied, the reference was normal 24-h ABPM. Hosmer-Lemeshow goodness of fit statistics were used to assess model fit. $P$ values less than 0.05 were considered statistically significant. Statistical analysis was performed with the SPSS version 22.0 package program for Windows (SPSS, Chicago, Illinois, USA).

\section{RESULTS}

Four-hundred fifty-six children with CKD stages III-V (64.3\% males, $71.3 \%$ CAKUT, mean age $12.5 \pm 3.2$ years, eGFR $29 \pm 12 \mathrm{ml} / \mathrm{min} / 1.73 \mathrm{~m}^{2}$ ) were included in the analysis. ABPM revealed normal BP throughout day and night in 
TABLE 1. Clinical characteristics of the patients according to ambulatory blood pressure monitoring groups

\begin{tabular}{|c|c|c|c|c|c|c|}
\hline \multirow[t]{2}{*}{ Patient characteristics } & \multicolumn{5}{|c|}{ ABPM groups } & \multirow[t]{2}{*}{$P$} \\
\hline & Normotension, $\boldsymbol{N}=294$ & INH, $N=61$ & IDH, $N=17$ & SH, $N=84$ & Total, $N=456$ & \\
\hline Male, $N(\%)$ & $194(66.0)$ & 39 (63.9) & $10(58.8)$ & $34(59.5)$ & $293(64.3)$ & $0.700^{*}$ \\
\hline Age (years) $^{* *}$ & $12.6(5.4)$ & $13.8(5.2)$ & $12.4(4.7)$ & $11.8(4.6)$ & $12.5(5.2)$ & $0.127^{* *}$ \\
\hline Height SDS ${ }^{* *}$ & $-1.32(1.84)$ & $-1.02(1.58)$ & $-1.50(1.69)$ & $-1.41(1.25)$ & $-1.32(1.64)$ & $0.110^{* *}$ \\
\hline BMI SDS** & $0.18(1.58)$ & $0.02(2.20)$ & $0.67(1.85)$ & $0.01(1.31)$ & $0.11(1.59)$ & $0.447^{* *}$ \\
\hline CKD duration (months) ${ }^{* *}$ & $70.4(91.0)^{a, b}$ & $44.6(85.3)^{a}$ & $59.7(85.1)$ & $41.6(57.9)^{b}$ & $58.6(85.6)$ & $<0.001$ \\
\hline \multicolumn{7}{|l|}{ Underlying disease, $N(\%)$} \\
\hline CAKUT & $216(73.5)$ & 39 (63.9) & $11(64.7)$ & $59(70.2)$ & $325(71.3)$ & $0.440^{*}$ \\
\hline Glomerulopathy & $18(6.1)$ & $2(3.3)$ & $1(5.9)$ & $6(7.1)$ & $27(5.9)$ & $0.793^{* * *}$ \\
\hline Post-AKI CKD & $14(4.8)$ & $2(3.3)$ & $1(5.9)$ & $2(2.4)$ & $19(4.2)$ & $0.687^{* * *}$ \\
\hline Tubulointerstitial diseases & $29(9.9)$ & $13(21.3)$ & $3(17.6)$ & $10(11.9)$ & $55(12.1)$ & $0.080^{*}$ \\
\hline Other or unknown & $24(8.2)$ & 7 (11.5) & $1(5.9)$ & $7(8.3)$ & $39(8.6)$ & $0.848^{*}$ \\
\hline \multicolumn{7}{|l|}{ Physical activity, $N(\%)$} \\
\hline $\mathrm{Oh} /$ week & $58 / 286(20.3)$ & $30 / 58(34.5)$ & $7 / 17(41.2)$ & $26 / 81(32.1)$ & $111 / 442(25.1)$ & \\
\hline $1-2 \mathrm{~h} /$ week & $47 / 286(16.4)$ & $8 / 58(13.8)$ & $0 / 17(0)$ & $10 / 81(12.3)$ & $65 / 442(14.7)$ & \\
\hline $3-4 \mathrm{~h} /$ week & 25/286 (8.7) & $5 / 58(8.6)$ & $2 / 17(11.8)$ & $6 / 81(7.4)$ & $38 / 442(8.6)$ & \\
\hline$>4 \mathrm{~h} /$ week & $156 / 286(54.5)$ & $25 / 58(43.1)$ & $8 / 17(47.1)$ & $39 / 81(48.1)$ & $228 / 442(51.6)$ & \\
\hline$\geq 1 \mathrm{~h} /$ week & $228 / 286(79.7)^{a, b}$ & $38 / 58(65.5)^{a}$ & $10 / 17(58.8)$ & $55 / 81(67.9)^{b}$ & $331 / 442(74.9)$ & $0.013^{*}$ \\
\hline \multicolumn{7}{|l|}{ CKD Stage, $n / N(\%)$} \\
\hline Stage III & $120 / 293(40.9)$ & 28/61 (45.9) & $7 / 15(41.2)$ & $27 / 84(32.2)$ & $182 / 455(40.0)$ & $0.371^{*}$ \\
\hline Stage IV & $157 / 293(53.6)$ & $29 / 61(47.5)$ & $8 / 15(47.1)$ & $44 / 84(52.4)$ & 238/455 (52.3) & $0.830^{*}$ \\
\hline Stage $\mathrm{V}$ & $16 / 293(5.5)^{\mathrm{b}}$ & $4 / 61(6.6)$ & $2 / 15(11.8)$ & $13 / 84(15.5)^{b}$ & $35 / 455(7.7)$ & $0.021^{* * *}$ \\
\hline eGFR $\left(\mathrm{ml} / \mathrm{min} \text { per } 1.73 \mathrm{~m}^{2}\right)^{* *}$ & $27.0(15.3)$ & $28.6(14.7)$ & $29.0(20.8)$ & $24.0(16.2)$ & $26.9(15.3)$ & $0.360^{* *}$ \\
\hline Albuminuria (mg/g creatinine) ${ }^{* *}$ & $264(857)^{a, b, c}$ & $487(1960)^{a}$ & $774(1157)^{c}$ & $1224(2401)^{b}$ & $368(1196)$ & $<0.001^{* *}$ \\
\hline
\end{tabular}

ABPM, ambulatory blood pressure monitoring; AKI, acute kidney injury; CAKUT, congenital anomalies of kidney and urinary tract; eGFR, estimated glomerular filtration rate; IDH, isolated daytime hypertension; INH, isolated nocturnal hypertension; IQR, interquartile range; SDS, SD score; SH, sustained hypertension.

Bold values represent a $P$ value $<0.05$.

${ }^{a}$ Statistically significant difference between normal and INH.

bStatistically significant difference between normal and SH.

'Statistically significant difference between normal and IDH.

${ }^{*}$ Chi-square test is used to compare four ABPM groups.

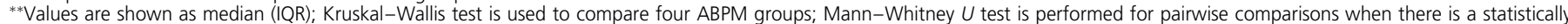

significant difference between four groups (main effect).
${ }_{* * * *}$ Fisher's exact test is used to compare four ABPM groups.

294 children (64.5\%), sustained hypertension in 84 children (18.4\%), INH in 61 children (13.4\%), and IDH in 17 children (3.7\%).

Three hundred and twenty-six patients (71.5\%) had normal casual BP; among those, the fractions of patients with normal ABPM, INH, IDH, and sustained hypertension were $74.8 \%(n=244), 14.4 \%(n=47), 2.5 \%(n=8)$, and 8.3\% ( $n=27)$, respectively (Supplement Table 1, http:// links.lww.com/HJH/B112). Accordingly, 82 children (18\%) had masked hypertension and 50 children (11\%) displayed white coat HTN, respectively.

The clinical characteristics of patients with normal ABPM, INH, and sustained hypertension are shown in Table 1. The groups were comparable for sex, age, height, BMI, underlying renal disease distribution, prevalence of prematurity, low birth weight, and smallness for gestational age (SGA). Median CKD vintage was significantly longer in the normal ABPM [70.4 (IQR 91.0) months; $P<0.001]$ compared with the INH [44.6 (IQR 85.3) months] and sustained hypertension patients [41.6 (IQR 57.9) months]. Physical activity at least $1 \mathrm{~h} /$ week was more common in children with normal ABPM; and CKD stage 5 was more common in the sustained hypertension group. Albuminuria was lower in the children with normal ABPM. The groups differed with respect to inorganic serum phosphorus, and PTH level, whereas $\mathrm{Hb}$, albumin, lipid, and hsCRP were comparable (Supplement Table 2, http://links.lww.com/ $\mathrm{HJH} / \mathrm{B} 112)$.
The mean daytime, night-time and 24-h mean arterial blood pressure (MAP) values, load and dipping and fraction of nondippers by group are presented in Table 2 and additional information for SBP and DBP variables are given in Supplement Table 3, http://links.lww.com/HJH/B112. Although the fraction of patients receiving antihypertensive therapy and the mean number of antihypertensive drugs were comparable, significantly more patients with sustained hypertension received calcium channel blockers compared with patients with controlled HTN (Table 2).

In the INH group, daytime BP was within the normal range, but significantly higher compared with normotensive patients (MAP SDS 0.60 vs. $-0.42, P<0.001$ ), by definition nocturnal BP was significantly elevated. In the IDH group, daytime BP was significantly elevated, but nocturnal BP did not differ from the normotensive group. Sustained hypertension patients had significantly higher day and night-time MAP SDS values compared with both IDH and INH (Table 2).

24-h BP load was lower in INH (41.2\%; probably due to the shorter night period; $6 \mathrm{~h})$ than in IDH patients $(59.2 \%$; $P=0.006)$; in both groups 24-h BP load was significantly lower compared with sustained hypertension (83.0\%; $P<0.001)$.

The fractions of patients receiving their antihypertensive medications during day or evening hours and the fractions of patients on single daily dose did not differ significantly between the ABPM phenotypes (Supplement Table 4, 
TABLE 2. Office blood pressure and ambulatory blood pressure monitoring findings and use of antihypertensive drugs of the patients according to ambulatory blood pressure monitoring groups

\begin{tabular}{|c|c|c|c|c|c|c|}
\hline \multirow[t]{2}{*}{ Parameters } & \multicolumn{5}{|c|}{ ABPM groups } & \multirow[t]{2}{*}{$\boldsymbol{P}$} \\
\hline & Normotension, $N=294$ & INH, $N=61$ & IDH, $N=17$ & $\mathrm{SH}, \boldsymbol{N}=\mathbf{8 4}$ & Total, $N=456$ & \\
\hline \multicolumn{7}{|l|}{ Office BP } \\
\hline Office SBP $(\mathrm{mmHg})^{*}$ & $110(17)^{a, b, c}$ & $110(17)^{a, d}$ & $120(20)^{b}$ & $120(18)^{c, d}$ & $110.0(20)$ & $<0.001^{*}$ \\
\hline Office SBP SDS* & $0.40(1.46)^{a, b, c}$ & $0.64(1.46)^{\mathrm{a}, \mathrm{d}, \mathrm{e}}$ & $1.57(1.80)^{\mathrm{b}, \mathrm{e}}$ & $2.03(1.62)^{c, d}$ & $0.65(1.73)$ & $<0.001^{*}$ \\
\hline Office SBP $>95$ th pct, $N(\%)$ & $41(14)^{b, c}$ & $12(20)^{d}$ & $7(41)^{b}$ & $51(62)^{c, d}$ & $111(24.3)$ & $<0.001^{* *}$ \\
\hline Office DBP $(\mathrm{mmHg})^{*}$ & $65.0(10)^{\mathrm{b}, \mathrm{c}}$ & $69.0(16)^{\mathrm{d}, \mathrm{e}}$ & $78.0(24)^{\mathrm{b}, \mathrm{e}}$ & $80.0(18)^{c, d}$ & $68(17)$ & $<0.001^{*}$ \\
\hline Office DBP SDS* & $0.23(1.05)^{a, b, c}$ & $0.38(1.13)^{a, d, e}$ & $1.33(2.06)^{\mathrm{b}, \mathrm{e}}$ & $1.72(1.47)^{c, d}$ & $0.49(1.41)$ & $<0.001^{*}$ \\
\hline Office DBP > 95th pct, $N(\%)$ & $24(8.2)^{b, c}$ & $6(9.8)^{d, e}$ & $7(41.2)^{\mathrm{b}, \mathrm{e}}$ & $44(52.4)^{c, d}$ & $81(17.8)$ & $<0.001^{* *}$ \\
\hline \multicolumn{7}{|l|}{ ABPM } \\
\hline Day MAP $(\mathrm{mmHg})^{*}$ & $83.8(10)^{a, b, c}$ & $91.2(6)^{a, d, e}$ & $98.0(7)^{b, e, f}$ & $102.9(11)^{c, d, f}$ & $88.1(13)$ & $<0.001^{*}$ \\
\hline Day MAP SDS* & $-0.42(1.33)^{a, b, c}$ & $0.60(0.91)^{a, d, e}$ & $1.83(1.07)^{b, e, f}$ & $2.67(1.91)^{c, d, f}$ & $0.22(2.0)$ & $<0.001^{*}$ \\
\hline Day MAP load (\%)* & $6.67(16)^{a, b, c}$ & $23.5(32)^{a, d, e}$ & $60.6(32)^{b, e}$ & $76.8(36)^{c, d}$ & $14.0(40)$ & $<0.001^{*}$ \\
\hline Night MAP $(\mathrm{mmHg})^{*}$ & $73.3(8)^{a, b, c}$ & $83.2(6)^{\mathrm{a}, \mathrm{d}, \mathrm{e}}$ & $77.3(3)^{\mathrm{b}, e, f}$ & $92.4(12)^{c, d, f}$ & $77.0(11)$ & $<0.001^{*}$ \\
\hline Night MAP SDS* & $0.39(1.33)^{a, b, c}$ & $1.97(0.87)^{a, d, e}$ & $1.01(0.47)^{b, e, f}$ & $3.09(1.83)^{c, d, f}$ & $0.91(1.87)$ & $<0.001^{*}$ \\
\hline Night MAP load (\%)* & $8.33(25)^{a, b, c}$ & $63.64(26)^{a, d, e}$ & $27.78(24)^{b, e, f}$ & $100.0(23)^{c, d, f}$ & $25(64)$ & $<0.001^{*}$ \\
\hline 24-h MAP $(\mathrm{mmHg})^{*}$ & $81.2(8)^{a, b, c}$ & $88.7(6)^{a, d, e}$ & $91.8(8)^{b, e, f}$ & $100.0(12)^{c, d, f}$ & $85.0(12)$ & $<0.001^{*}$ \\
\hline 24-h MAP SDS* & $-0.12(1.33)^{a, b, c}$ & $1.18(0.86)^{\mathrm{a}, \mathrm{d}, \mathrm{e}}$ & $1.72(1.25)^{\mathrm{b}, \mathrm{e}, \mathrm{f}}$ & $3.28(1.97)^{c, d, f}$ & $0.52(2.06)$ & $<0.001^{*}$ \\
\hline 24-h MAP load $(\%)^{*}$ & $13.5(22)^{a, b, c}$ & $41.2(24)^{a, d, e}$ & $59.2(25)^{b, e, f}$ & $83.0(28)^{c, d, f}$ & $27.3(46)$ & $<0.001^{*}$ \\
\hline MAP dipping $(\mathrm{mmHg})^{* * * *}$ & $12.9 \pm 5.7^{a, b, c}$ & $7.1 \pm 4.7^{\mathrm{a}, \mathrm{d}, \mathrm{e}}$ & $22.0 \pm 3.4^{\mathrm{b}, e, f}$ & $10.8 \pm 6.8^{c, d, f}$ & $12.1 \pm 6.3$ & $<0.001^{* * *}$ \\
\hline MAP nondipper, $N(\%)$ & $86(29.3)^{a, b}$ & $42(68.9)^{\mathrm{a}, \mathrm{d}, \mathrm{e}}$ & $0(0)^{b, e, f}$ & $34(40.5)^{d, f}$ & $162(35.5)$ & $<0.001^{* *}$ \\
\hline Number of $\mathrm{AHT}^{*}$ & $0(1)$ & $0(1)$ & $0(1)$ & $0(1)$ & $0(1)$ & $0.818^{*}$ \\
\hline AHTs (yes), N (\%) & $126(42.9)$ & $26(42.6)$ & $7(41.2)$ & $41(48.8)$ & $200(43.9)$ & $0.791^{* *}$ \\
\hline ACE-i & $99(33.7)$ & $17(27.9)$ & $5(29.4)$ & $20(23.8)$ & $141(30.9)$ & $0.345^{* *}$ \\
\hline ARB & $23(7.8)$ & $3(4.9)$ & $1(5.9)$ & $3(3.6)$ & $30(6.6)$ & $0.534^{* * * *}$ \\
\hline CCB & $31(10.5)^{c}$ & $11(18.0)$ & $3(17.6)$ & $24(28.6)^{c}$ & $69(15.1)$ & $0.001^{* *}$ \\
\hline Beta blockers & $8(2.7)$ & $4(6.6)$ & $0(0)$ & $4(4.8)$ & $16(3.5)$ & $0.349^{* * * * *}$ \\
\hline
\end{tabular}

ABPM, ambulatory blood pressure monitoring; ACE-i, angiotensin converting enzyme inhibitors; AHT, antihypertensive drug; ARB, AT1 blockers; CCB, calcium channel blockers; INH, isolated nocturnal hypertension; IQR, interquartile range; MAP, mean arterial blood pressure; SDS, SD score; SH, sustained hypertension.

Bold values represent a $P$ value $<0.05$.

aStatistically significant difference between normal and INH.

bstatistically significant difference between normal and IDH.

'Statistically significant difference between normal and SH.

dStatistically significant difference between INH and SH.

eStatistically significant difference between INH and IDH.

Statistically significant difference between IDH and $\mathrm{SH}$.

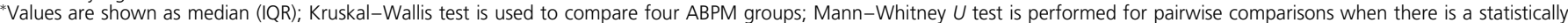

significant difference between four groups (main effect).
${ }^{* *}$ Chi-square test is used to compare four ABPM groups.

${ }^{* * * *}$ Vhalues are shown as mean \pm SD; one-way analysis of variance (ANOVA) test is used to compare four ABPM groups; $t$ test is performed for pairwise comparisons when there is a statistically significant difference between four groups (main effect).

****Fisher's exact test is used to compare four ABPM groups.

http://links.lww.com/HJH/B112). Load and dipping data for daytime, night-time and 24-h were comparable among treated and untreated normotensive patients (data not shown).

Presence of SGA, older age, higher height SDS and PTH, and shorter duration of CKD were independent determinants of INH (Table 3).

\section{Cardiovascular measures}

PWV SDS, CIMT SDS, and LVMI were higher in sustained hypertension compared with patients with normal ABPM

TABLE 3. Independent risk factors for isolated nocturnal hypertension (reference: normotension)

\begin{tabular}{|lccc|}
\hline Risk factors & OR & $\mathbf{9 5} \% \mathbf{C l}$ & $\boldsymbol{P}$ \\
\hline Age (year) & 1.148 & {$[1.030 ; 1.280]$} & 0.013 \\
\hline SGA (vs. normal) & 2.613 & {$[1.194 ; 5.717]$} & 0.016 \\
\hline Height SDS & 1.507 & {$[1.149 ; 1.976]$} & 0.003 \\
\hline CKD duration (months) & 0.992 & {$[0.986 ; 0.998]$} & 0.010 \\
\hline Office SBP SDS & 1.285 & {$[0.995 ; 1.660]$} & 0.055 \\
\hline PTH (pmol/l) & 1.012 & {$[1.001 ; 1.023]$} & 0.027 \\
\hline
\end{tabular}

$\mathrm{Cl}$, confidence interval; $\mathrm{CKD}$, chronic kidney disease; OR, odds ratio; $\mathrm{PTH}$, parathyroid hormone; SDS, SD score; SGA, smallness for gestational age.
$(P<0.001)$ (Table 4, Fig. $1 \mathrm{a}-\mathrm{c})$. In patients with INH PWV SDS and cIMT SDS were also higher compared with normal ABPM $(P<0.05)$, whereas the difference in LVMI did not reach statistical significance.

A subgroup analysis considering the use of antihypertensive agents (AHT) did not show any significant effect on cardiovascular measures in general, but PWV SDS in the normal ABPM group with AHT was significantly lower compared with the normal ABPM group without AHT $(-0.51 \pm 1.64$ vs. $0.15 \pm 1.39 ; P<0.001$; Supplement Table 5, http://links.lww.com/HJH/B112).

BP pattern over $24 \mathrm{~h}$ correlated with the cumulative number of cardiovascular abnormalities (Fig. 2; $r=0.275$, $P<0.001)$. The fraction of patients with two or more cardiovascular abnormalities was higher in the INH group (14/42; $33.3 \%)$ compared with patients with normal ABPM findings (36/215; 16.7\%; $P=0.013)$, but lower than in sustained hypertension patients $(24 / 57 ; 42.1 \% ; P=0.375)$. The fraction of patients with two or more cardiovascular abnormalities was not significantly different in the IDH group (4/16; 25.0\%) compared with patients with normal ABPM findings (36/215; $16.7 \% ; P=0.40$, Fisher's exact test), and in sustained hypertension patients $(24 / 57 ; 42.1 \% ; P=0.214)$. 
TABLE 4. Cardiovascular measures according to ambulatory blood pressure monitoring groups

\begin{tabular}{|c|c|c|c|c|c|}
\hline & \multicolumn{4}{|c|}{ ABPM groups } & \multirow[t]{2}{*}{$\boldsymbol{P}$} \\
\hline & Normal & INH & IDH & SH & \\
\hline $\operatorname{PWV}(\mathrm{m} / \mathrm{s})^{*}$ & $4.67(1.00)^{a, b, c}(n=223)$ & $4.93(1.00)^{\mathrm{a}}(n=45)$ & $4.97(1.00)^{\mathrm{b}}(n=16)$ & $5.21(1.00)^{c}(n=62)$ & $<0.001^{*}$ \\
\hline PWV SDS** & $-0.13 \pm 1.54^{a, b, c}(n=223)$ & $0.55 \pm 1.42^{\mathrm{a}, \mathrm{d}}(n=45)$ & $0.85 \pm 1.51^{\mathrm{b}}(n=16)$ & $1.38 \pm 1.94^{\mathrm{c}, \mathrm{d}}(n=62)$ & $<0.001^{* *}$ \\
\hline Elevated PWV, $N(\%)$ & $27 / 223(12.1)^{a, c}$ & $11 / 45(24.4)^{\mathrm{a}, \mathrm{d}}$ & $4 / 16(25.0)$ & $30 / 62(48.4)^{c, d}$ & $<0.001^{* * *}$ \\
\hline $\mathrm{CIMT}(\mathrm{mm})^{*}$ & $0.45(0.08)^{\mathrm{a}, \mathrm{c}}(n=225)$ & $0.47(0.08)^{a}(n=44)$ & $0.47(0.07)(n=16)$ & $0.46(0.09)^{c}(n=60)$ & $0.012^{*}$ \\
\hline CIMT SDS* & $1.44(1.60)^{\mathrm{a}, \mathrm{c}}(n=225)$ & $1.72(1.67)^{\mathrm{a}}(n=44)$ & $1.71(1.70)(n=16)$ & $1.94(2.06)^{c}(n=60)$ & $0.001^{*}$ \\
\hline Elevated cIMT, $N(\%)$ & $93 / 225(41.3)$ & $25 / 44(56.8)$ & $8 / 16(50.0)$ & $33 / 60(55.0)$ & $0.104^{* * *}$ \\
\hline $\operatorname{LVM}(\mathrm{g})^{*}$ & $87.5(45.1)(n=223)$ & $96.8(53.7)(n=45)$ & $99.0(44.9)(n=16)$ & $85.2(52.4)(n=63)$ & $0.313^{*}$ \\
\hline LVMI $\left[g /\left(m^{2.16}+0.09\right)\right]^{*}$ & $39.7(15.8)^{c}$ & $41.2(20.1)$ & $44.1(17.8)$ & $47.4(19.4)^{c}$ & $<0.001^{*}$ \\
\hline LV hypertrophy, $N(\%)$ & $71 / 223(31.8 \%)^{c}$ & $19 / 45(42.2)$ & $7 / 16(43.8)$ & $36 / 63(57.1)^{c}$ & $0.003^{* * *}$ \\
\hline
\end{tabular}

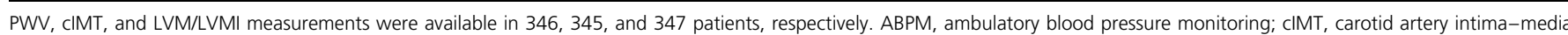
thickness; IDH, isolated daytime hypertension; IQR, interquartile range; INH, isolated nocturnal hypertension; LVM, left ventricular mass; LVMI, left ventricular mass index; PWV, pulse wave velocity; $\mathrm{SH}$, sustained hypertension.

Bold values represent a $P$ value $<0.05$.

${ }^{\text {aS }}$ tatistically significant difference between normal and $\mathrm{INH}$.

bstatistically significant difference between normal and IDH.

'Statistically significant difference between normal and $\mathrm{SH}$.

${ }^{d}$ Statistically significant difference between INH and SH.

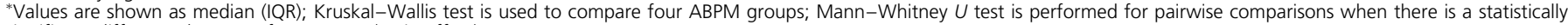
significant difference between four groups (main effect).

${ }^{* *}$ Values are shown as mean \pm SD; one-way analysis of variance (ANOVA) test is used to compare four ABPM groups; $t$ test is performed for pairwise comparisons when there is a statistically significant difference between four groups (main effect).

****Chi-square test is used to compare four ABPM groups.

In multivariable analyses, night-time HTN (Model A) and type of ABPM profiles (Model B) remained significantly associated with left ventricular hypertrophy (LVH), elevated cIMT and elevated PWV (Table 5). Nocturnal HTN independently predicted the risk of LVH and elevated cIMT and PWV.

Presence of sustained hypertension was predictive for LVH and higher PWV, while INH was predictive for the risk of elevated cIMT only and IDH was not predictive for cardiovascular risk in the logistic regression model.

\section{DISGUSSION}

This is the first study to compare the prevalence and relationship of INH and IDH with cardiovascular morbidity in children with predialysis CKD stages III-V. Sustained hypertension, INH and IDH were identified in 18.4, 13.4 and $3.7 \%$ of patients, respectively. In the general adult population INH has been reported in $6-11 \%$ and IDH in 5-14\%, with slightly higher rates observed among Europeans compared with Asians and Africans [10]. The prevalence of INH and IDH among healthy children is unknown.
In adults with CKD, a recent study from China reported sustained hypertension, INH and IDH prevalence of 50.8, 20.4 and $1.5 \%$, respectively [34]. Hence, the prevalence of INH in children with CKD appears to range between the general adult population and adult CKD patients. The lower frequency of IDH observed in CKD patients compared with the general population is probably attributable to their higher frequency of INH and sustained hypertension.

We identified by multivariate analysis a history of SGA, older age, shorter duration of CKD, higher height SDS and hyperparathyroidism as risk factors for INH. The observed association of SGA with INH is in keeping with previous findings in pediatric non-CKD populations and suggests that a selective upregulation of nocturnal BP may be an early sign of the adverse cardiovascular phenotype resulting from reprogramming in response to adverse in-utero conditions $[35,36]$. Older age, larger standardized height and shorter CKD duration might be related to non-CAKUT diseases, which usually show faster CKD progression. Higher PTH levels are associated with an increased risk for HTN even within the normal PTH range [37]. PTH may increase BP via activation of the renin-angiotensin-aldosterone system,
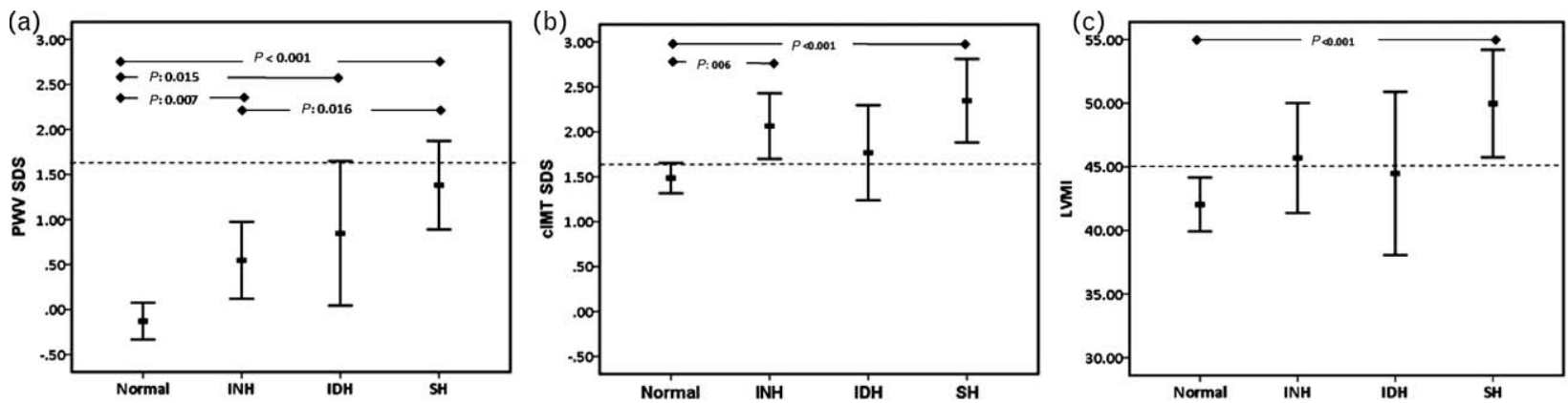

FIGURE 1 Mean pulse wave velocity SD score (a), carotid intima-media thickness SD score (b) and left ventricular mass index (c) values by ambulatory blood pressure monitoring groups. Error bars represent \pm 2 SEM. Dashed lines represent 95 th percentile values for pulse wave velocity SD score and carotid intima-media thickness SD score, and $45 \mathrm{~g} / \mathrm{m}^{2.16}$ for left ventricular mass index, respectively. ABPM, ambulatory blood pressure monitoring; IDH, isolated daytime hypertension; INH, isolated nocturnal hypertension; SH, sustained hypertension. 


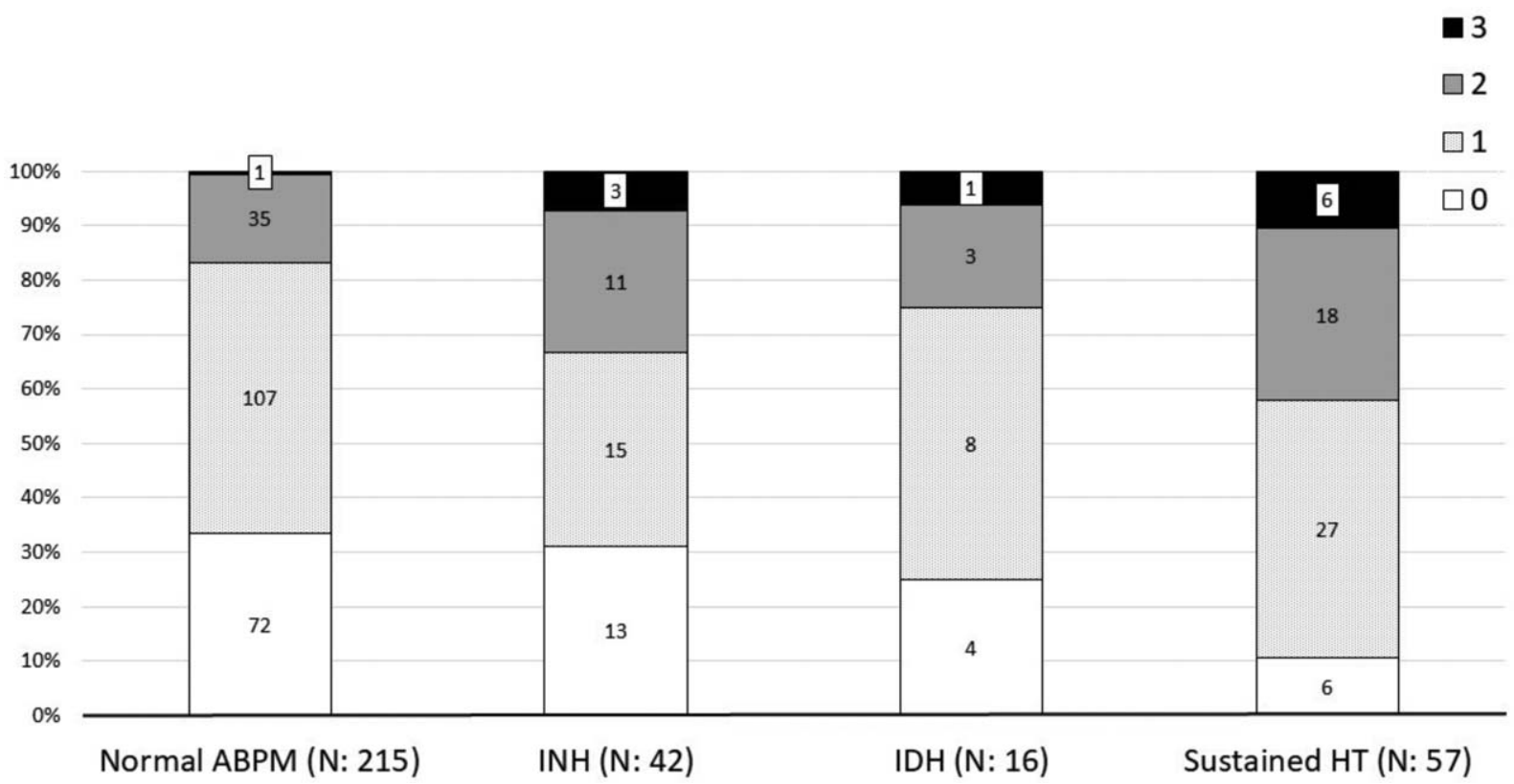

FIGURE 2 Percentages and numbers of patients presenting with zero, one, two, or three abnormal cardiovascular measures by ambulatory blood pressure monitoring groups. ABPM, ambulatory blood pressure monitoring; HT, hypertension; IDH, isolated daytime hypertension; INH, isolated nocturnal hypertension.

TABLE 5. Logistic regression models for cardiovascular outcome (Model A included day/night hypertension; Model B included type of ambulatory blood pressure monitoring profiles, see definitions and statistical analysis for details)

\begin{tabular}{|c|c|c|c|c|c|c|c|c|c|}
\hline \multirow[t]{2}{*}{ Variables } & \multicolumn{3}{|c|}{ LVH } & \multicolumn{3}{|c|}{ Elevated cIMT } & \multicolumn{3}{|c|}{ Elevated PWV } \\
\hline & OR & $95 \% \mathrm{Cl}$ & $P$ & OR & $95 \% \mathrm{Cl}$ & $P$ & OR & $95 \% \mathrm{Cl}$ & $\boldsymbol{P}$ \\
\hline Male sex & 2.298 & $1.338 ; 3.944$ & 0.003 & & & & 0.454 & $0.241 ; 0.855$ & 0.015 \\
\hline Low birth weight & & & & 2.371 & $1.130 ; 4.976$ & 0.022 & & & \\
\hline BMI SDS & 1.748 & $1.388 ; 2.200$ & $<0.001$ & 1.292 & $1.049 ; 1.592$ & 0.016 & & & \\
\hline Physical activity $\geq 3 \mathrm{~h} /$ week & 0.554 & $0.333 ; 0.921$ & 0.023 & 1.965 & $1.178 ; 3.278$ & 0.010 & & & \\
\hline Hemoglobin (g/dl) & & & & 0.828 & $0.706 ; 0.971$ & 0.020 & & & \\
\hline Phosphate (mmol/l) & & & & & & & 0.309 & $0.123 ; 0.780$ & 0.013 \\
\hline LDL (mg/dl) & & & & & & & 1.010 & $1.001 ; 1.019$ & 0.029 \\
\hline eGFR $\left(\mathrm{ml} / \mathrm{min}\right.$ per $\left.1.73 \mathrm{~m}^{2}\right)$ & 0.969 & $0.947 ; 0.992$ & 0.009 & & & & & & \\
\hline Office-SBP SDS & & & & & & & 1.764 & $1.388 ; 2.243$ & $<0.001$ \\
\hline Daytime HT & & & NS & & & NS & & & NS \\
\hline Night-time HT & 2.458 & $1.434 ; 4.214$ & 0.001 & 2.016 & $1.125 ; 3.614$ & 0.018 & 2.959 & $1.561 ; 5.608$ & 0.001 \\
\hline \multicolumn{10}{|l|}{ Model B } \\
\hline \multirow[t]{2}{*}{ Variables } & \multicolumn{3}{|c|}{ LVH } & \multicolumn{3}{|c|}{ Elevated cIMT } & \multicolumn{3}{|c|}{ Elevated PWV } \\
\hline & OR & $95 \% \mathrm{Cl}$ & $P$ & OR & $95 \% \mathrm{Cl}$ & $P$ & OR & $95 \% \mathrm{Cl}$ & $\boldsymbol{P}$ \\
\hline Male sex & 2.379 & $1.377 ; 4.112$ & 0.002 & & & & 0.466 & $0.246 ; 0.883$ & 0.019 \\
\hline Low birth weight & & & & 2.491 & $1.178 ; 5.267$ & 0.017 & & & \\
\hline BMI SDS & 1.726 & $1.370 ; 2.175$ & $<0.001$ & 1.290 & $1.045 ; 1.592$ & 0.018 & & & \\
\hline Physical activity $\geq 3 \mathrm{~h} /$ week & 0.551 & $0.330 ; 0.919$ & 0.022 & 1.982 & $1.184 ; 3.319$ & 0.009 & & & \\
\hline Hemoglobin (g/dl) & & & & 0.827 & $0.705 ; 0.971$ & 0.020 & & & \\
\hline Phosphate (mmol/l) & & & & & & & 0.272 & $0.105 ; 0.707$ & 0.008 \\
\hline LDL (mg/dl) & & & & & & & 1.009 & $1.000 ; 1.018$ & 0.053 \\
\hline eGFR (ml/min per $\left.1.73 \mathrm{~m}^{2}\right)$ & 0.969 & $0.947 ; 0.992$ & 0.009 & & & & & & \\
\hline Office-SBP SDS & & & & & & & 1.661 & $1.296 ; 2.128$ & $<0.001$ \\
\hline ABPM type (ref: normotension) & & & 0.005 & & & 0.051 & & & 0.004 \\
\hline ABPM-INH & 1.820 & $0.868 ; 3.815$ & 0.113 & 2.832 & $1.170 ; 6.859$ & 0.021 & 2.049 & $0.846 ; 4.962$ & 0.112 \\
\hline ABPM-IDH & 1.662 & $0.521 ; 5.295$ & 0.391 & 2.074 & $0.672 ; 6.399$ & 0.205 & 1.662 & $0.436 ; 6.336$ & 0.457 \\
\hline ABPM-SH & 3.281 & $1.682 ; 6.399$ & $<0.001$ & 1.802 & $0.895 ; 3.626$ & 0.099 & 4.428 & $1.985 ; 9.879$ & $<0.001$ \\
\hline
\end{tabular}

ABPM, ambulatory blood pressure monitoring; cIMT, carotid artery intima-media thickness; eGFR, estimated glomerular filtration rate; $\mathrm{HT}$, hypertension; IDH, isolated daytime hypertension; INH, isolated nocturnal hypertension; LVH, left ventricular hypertrophy; OR, odds ratio; PTH, parathyroid hormone; PWV, pulse wave velocity; SDS, SD score; SH, sustained hypertension. 
impairment of endothelial vasodilatory function and thickening of arterial vessel through its prosclerotic effect on smooth muscle cells [38-40].

Studies in adults have provided solid evidence that INH is associated with cardiovascular risk [11-14]. In a recent meta-analysis including 3657 adult patients, both LVMI and cIMT were higher in patients with nocturnal HTN than in those with nocturnal normotension [41].

Data for children and adolescents is limited. In a study of 82 pediatric patients with type 1 diabetes nocturnal HTN, present in 39\% of patients, was associated with increased CIMT [42].

Here we demonstrate for the first time that both PWV and CIMT are higher in children with CKD and INH, compared with those with normal ambulatory BP. The associations of nocturnal HTN with these intermediate cardiovascular endpoints were independent of other clinical and biochemical risk factors. In addition, our study suggests that not only INH but also IDH may associate with markers of early cardiovascular morbidity. Despite its low prevalence, IDH was associated with higher PWV SDS. Likewise, CIMT SDS and LVMI were nominally higher compared with the normotensive group although statistical significance was not reached probably due to the small IDH sample size. Finally, we found albuminuria to be equally elevated with INH, IDH and sustained hypertension as compared with normotensive patients. Taken together, these observations suggest that BP elevation, regardless of the time of day, sustained or not, is a risk factor for the development of target-organ lesions.

It has been speculated that adequate treatment of INH normalizing night-time BP level and restoring nocturnal dipping pattern might have an impact on albuminuria, renal disease progression, and cardiovascular risk. Hermida et al. [43] showed that among adult patients with CKD and HTN, taking at least one antihypertensive medication at bedtime improved nocturnal BP control and reduced the risk for cardiovascular events. In our cohort, antihypertensive therapy (medication, dosage, time of medications) was at the discretion of the investigators; we were not able to show an impact of taking at least one antihypertensive medication at bedtime on INH. A prospective, randomized study would be needed to elucidate this question.

Although the major strength of this study is the large number of patients collected in a large multicenter, multinational effort with standardized methodology, the analysis presented here is limited by its cross-sectional design and the diagnosis of INH based on single ABPM profiles. Limited published information is available regarding the reproducibility of INH. In a short-term study, INH showed higher persistence than the diagnosis of a blunted nocturnal BP decline (nondipping) [44]. In a long-term study of 30 patients, INH persisted in 10 individuals whereas 10 subjects developed sustained HTN, two shifted to IDH, and eight became normotensive [10]. Furthermore, the findings of our study might have been influenced by antihypertensive therapy, applied in $44 \%$ of the patients, which might attenuate the association of HTN with cardiovascular outcome. Finally, the number of patients with IDH was relatively small and it was not possible to make a subgroups analysis, that is compare INH and IDH, to suggest whether there is a differential effect of night-time hemodynamics on myocardium and vasculature. Cross sectional design of the study did not allow to test whether the changes in PWV and cIMT precede an increase in LVMI.

In conclusion, we demonstrate that the prevalence of INH and IDH in children with CKD prior to dialysis were 13.4 and $3.7 \%$, respectively. INH was independently associated with elevated cIMT; elevated night-time BP in general was associated with elevated PWV, CIMT, and LVH. Whether the changes in PWV and CIMT precede an increase in LVMI and whether adequate treatment of INH might prevent cardiovascular morbidity and renal disease progression remains to be shown by longitudinal data analysis.

\section{AGKNOWLEDGEMENTS}

Support for the 4C Study was received from the ERA-EDTA Research Programme, the KfH Foundation for Preventive Medicine and the German Federal Ministry of Education and Research (reference number: 01EO0802). F.S. and M.K. received support for this study from the European Community's Seventh Framework Programme (FP7/2007-2013) under grant agreement No. 2012-305608 (EURenOmics). A.D. received support from the Turkish Academy of Sciences (Programme to Reward Successful Young Scientists, TUBAGEBIP/2006-6) and the Turkish Council of Higher Education. Several authors of this publication (K.A., R.S., A.B., B.R., A.J., A.M., F.S., E.W.) are members of the European Reference Network for Rare Kidney Diseases (ERKNet).

\section{Conflicts of interest}

There are no conflicts of interest.

\section{REFERENGES}

1. Kavey RE, Allada V, Daniels SR, Hayman LL, McCrindle BW, Newburger JW, et al. Cardiovascular risk reduction in high-risk pediatric patients. Circulation 2006; 114:2710-2738.

2. Foley RN, Parfrey PS, Sarnak MJ. Clinical epidemiology of cardiovascular disease in chronic renal disease. Am J Kidney Dis 1998; 32 (5 Suppl. 3):S112-S119.

3. Klag MJ, Whelton PK, Randall BL, Neaton JD, Brancati FL, Stamler J. End-stage renal disease in African-American and white men. 16-year MRFIT findings. JAMA 1997; 277:1293-1298.

4. Oldrizzi L, Rugiu C, De Biase V, Maschio G. The place of hypertension among the risk factors for renal function in chronic renal failure. $A m J$ Kidney Dis 1993; 21 (5 Suppl. 2):119-123.

5. Wingen AM, Fabian-Bach C, Schaefer F, Mehls O. Randomised multicentre study of a low-protein diet on the progression of chronic renal failure in children. European Study Group of Nutritional Treatment of Chronic Renal Failure in Childhood. Lancet 1997; 349:1117-1123.

6. Mitsnefes M, Ho PL, McEnery PT. Hypertension and progression of chronic renal insufficiency in children: a report of the North American Pediatric Renal Transplant Cooperative Study (NAPRTCS). J Am Soc Nephrol 2003; 14:2618-2622.

7. Flynn JT, Mitsnefes M, Pierce C, Cole SR, Parekh RS, Furth SL, et al., Chronic Kidney Disease in Children Study Group. Blood pressure in children with chronic kidney disease: a report from the Chronic Kidney Disease in Children study. Hypertension 2008; 52:631-637.

8. Wühl E, Schaefer F, Mehls O. Prevalence and current treatment policies of hypertension and proteinuria in children with chronic renal failure in Europe. In: Timio M, Wizemann V, Venanzi S, editors. Cardionephrology. Cosenza: Editoriale Bios; 1999. pp. 85-88.

9. Agarwal R, Andersen MJ. Prognostic importance of ambulatory blood pressure recordings in patients with chronic kidney disease. Kidney Int 2006; 69:1175-1180. 
10. Li Y, Staessen JA, Lu L, Li LH, Wang GL, Wang JG. Is isolated nocturnal hypertension a novel clinical entity? Findings from a Chinese population study. Hypertension 2007; 50:333-339.

11. Hoshide S, Ishikawa J, Eguchi K, Ojima T, Shimada K, Kario K. Masked nocturnal hypertension and target organ damage in hypertensives with well-controlled self-measured home blood pressure. Hypertens Res 2007; 30:143-149.

12. Wijkman M, Länne T, Engvall J, Lindström T, Ostgren CJ, Nystrom FH. Masked nocturnal hypertension - a novel marker of risk in type 2 diabetes. Diabetologia 2009; 52:1258-1264.

13. Ogedegbe G, Spruill TM, Sarpong DF, Agyemang C, Chaplin W, Pastva A, et al. Correlates of isolated nocturnal hypertension and target organ damage in a population-based cohort of African Americans: the Jackson Heart Study. Am J Hypertens 2013; 26:1011-1016.

14. Fan HQ, Li Y, Thijs L, Hansen TW, Boggia J, Kikuya M, et al., International Database on Ambulatory Blood Pressure In Relation to Cardiovascular Outcomes Investigators. Prognostic value of isolated nocturnal hypertension on ambulatory measurement in 8711 individuals from 10 populations. J Hypertens 2010; 28:2036-2045.

15. Lurbe E, Redon J, Kesani A, Pascual JM, Tacons J, Alvarez V, et al. Increase in nocturnal blood pressure and progression to microalbuminuria in type 1 diabetes. $N$ Engl J Med 2002; 347:797-805.

16. Lurbe E, Torro I, Alvarez V, Nawrot T, Paya R, Redon J, et al. Prevalence, persistence, and clinical significance of masked hypertension in youth. Hypertension 2005; 45:493-498.

17. Querfeld U, Anarat A, Bayazit AK, Bakkaloglu A, Bilginer Y, Caliskan S, et al., 4C Study Group. The cardiovascular comorbidity in children with chronic kidney disease (4C) study: objectives, design, and methodology. Clin J Am Soc Nephrol 2010; 5:1642-1648.

18. Schaefer F, Doyon A, Azukaitis K, Bayazit A, Canpolat N, Duzova A, et al., 4C Study Consortium. Cardiovascular phenotypes in children with CKD: the 4C study. Clin J Am Soc Nephrol 2017; 12:19-28.

19. The WHO Child Growth Standards. Available at: www.who.int/childgrowth/standards/en. [Accessed 14 February 2014].

20. National High Blood Pressure Education Program Working Group on High Blood Pressure in Children and Adolescents. The fourth report on the diagnosis, evaluation, and treatment of high blood pressure in children and adolescents. Pediatrics 2004; 114 (2 Suppl. 4th Report):555-576.

21. Doyon A, Fischer DC, Bayazit AK, Canpolat N, Duzova A, Sözeri B, et al., 4C Study Consortium. Markers of bone metabolism are affected by renal function and growth hormone therapy in children with chronic kidney disease. PLoS One 2015; 10:e113482.

22. Schwartz GJ, Muñoz A, Schneider MF, Mak RH, Kaskel F, Warady BA, et al. New equations to estimate GFR in children with CKD. J Am Soc Nephrol 2009; 20:629-637.

23. Hogg RJ, Furth S, Lemley KV, Portman R, Schwartz GJ, Coresh J, et al., National Kidney Foundation's Kidney Disease Outcomes Quality Initiative. National Kidney Foundation's Kidney Disease Outcomes Quality Initiative clinical practice guidelines for chronic kidney disease in children and adolescents: evaluation, classification, and stratification. Pediatrics 2003; 111 (6 Pt. 1):1416-1421.

24. Wühl E, Witte K, Soergel M, Mehls O, Schaefer F, German Working Group on Pediatric Hypertension. Distribution of 24-h ambulatory blood pressure in children: normalized reference values and role of body dimensions. J Hypertens 2002; 20:1995-2007.

25. Urbina E, Alpert B, Flynn J, Hayman L, Harshfield GA, Jacobson M, et al., American Heart Association Atherosclerosis, Hypertension, and Obesity in Youth Committee. ABPM in children and adolescents: recommendations for standard assessment: a scientific statement from the American Heart Association Atherosclerosis, Hypertension and Obesity in Youth Committee of the Council on Cardiovascular Disease in the Young and the Council for High Blood Pressure Research. Hypertension 2008; 52:433-451.

26. Parati G, Stergiou G, O'Brien E, Asmar R, Beilin L, Bilo G, et al., European Society of Hypertension Working Group on Blood Pressure Monitoring and Cardiovascular Variability. European Society of Hypertension practice guidelines for ambulatory blood pressure monitoring. J Hypertens 2014; 32:1359-1366.
27. Flynn JT, Daniels SR, Hayman LL, Maahs DM, McCrindle BW, Mitsnefes M, et al., American Heart Association Atherosclerosis, Hypertension and Obesity in Youth Committee of the Council on Cardiovascular Disease in the Young. Update: ambulatory blood pressure monitoring in children and adolescents: a scientific statement from the American Heart Association. Hypertension 2014; 63:1116-1135.

28. Devereux RB, Alonso DR, Lutas EM, Gottlieb GJ, Campo E, Sachs I, et al. Echocardiographic assessment of left ventricular hypertrophy: comparison to necropsy findings. Am J Cardiol 1986; 57:450-458.

29. Chinali M, Emma F, Esposito C, Rinelli G, Franceschini A, Doyon A, et al. Left ventricular mass indexing in infants, children, and adolescents: a simplified approach for the identification of left ventricular hypertrophy in clinical practice. J Pediatr 2016; 170:193-198.

30. Touboul PJ, Hennerici MG, Meairs S, Adams H, Amarenco P, Bornstein $\mathrm{N}$, et al. Mannheim carotid intima-media thickness and plaque consensus (2004-2006-2011). An update on behalf of the advisory board of the 3rd, 4th and 5th watching the risk symposia, at the 13th, 15th and 20th European Stroke Conferences, Mannheim, Germany, 2004, Brussels, Belgium, 2006, and Hamburg, Germany, 2011. Cerebrovasc Dis 2012; 34:290-296.

31. Doyon A, Kracht D, Bayazit AK, Deveci M, Duzova A, Krmar RT, et al., $4 \mathrm{C}$ Study Consortium. Carotid artery intima-media thickness and distensibility in children and adolescents: reference values and role of body dimensions. Hypertension 2013; 62:550-556.

32. Kracht D, Shroff R, Baig S, Doyon A, Jacobi C, Zeller R, et al. Validating a new oscillometric device for aortic pulse wave velocity measurements in children and adolescents. Am J Hypertens 2011; 24:12941299.

33. Thurn D, Doyon A, Sözeri B, Bayazit AK, Canpolat N, Duzova A, et al., $4 \mathrm{C}$ Study Consortium. Aortic pulse wave velocity in healthy children and adolescents: reference values for the vicorder device and modifying factors. Am J Hypertens 2015; 28:1480-1488.

34. Wang C, Deng WJ, Gong WY, Zhang J, Tang H, Peng H, et al. High prevalence of isolated nocturnal hypertension in Chinese patients with chronic kidney disease. J Am Heart Assoc 2015; 4:e002025.

35. Bayrakci US, Schaefer F, Duzova A, Yigit S, Bakkaloglu A. Abnormal circadian blood pressure regulation in children born preterm.J Pediatr 2007; 151:399-403.

36. Wolfenstetter A, Simonetti GD, Pöschl J, Schaefer F, Wühl E. Altered cardiovascular rhythmicity in children born small for gestational age. Hypertension 2012; 60:865-870.

37. Taylor EN, Curhan GC, Forman JP. Parathyroid hormone and the risk of incident hypertension. J Hypertens 2008; 26:1390-1394.

38. Gennari C, Nami R, Gonnelli S. Hypertension and primary hyperparathyroidism: the role of adrenergic and renin-angiotensin-aldosterone systems. Miner Electrolyte Metab 1995; 21:77-81.

39. Kosch M, Hausberg M, Vormbrock K, Kisters K, Gabriels G, Rahn KH, et al. Impaired flow-mediated vasodilation of the brachial artery in patients with primary hyperparathyroidism improves after parathyroidectomy. Cardiovasc Res 2000; 47:813-818.

40. Perkovic V, Hewitson TD, Kelynack KJ, Martic M, Tait MG, Becker GJ. Parathyroid hormone has a prosclerotic effect on vascular smooth muscle cells. Kidney Blood Press Res 2003; 26:27-33.

41. Cuspidi C, Sala C, Tadic M, Gherbesi E, Grassi G, Mancia G. Nocturnal hypertension and subclinical cardiac and carotid damage: an updated review and meta-analysis of echocardiographic studies. Clin Hypertens (Greenwich) 2016; 18:913-920.

42. Lee SH, Kim JH, Kang MJ, Lee YA, Won Yang S, Shin CH. Implications of nocturnal hypertension in children and adolescents with type 1 diabetes. Diabetes Care 2011; 34:2180-2185.

43. Hermida RC, Ayala DE, Mojon A, Fernandez JR. Bedtime dosing of antihypertensive medications reduces cardiovascular risk in CKD. J Am Soc Nephrol 2011; 22:2313-2321.

44. White WB, Larocca GM. Improving the utility of the nocturnal hypertension definition by using absolute sleep blood pressure rather than the "dipping" proportion. Am J Cardiol 2003; 92:1439-1441. 